\title{
Immune Monitoring for Advanced Cell Therapy Trials in Transplantation: Which Assays and When?
}

\author{
Helen L. Stark ${ }^{1}$, Hayson C. Wang ${ }^{1,2}$, Jasmina Kuburic ${ }^{1}$, Alaa Alzhrani ${ }^{1}$, Joanna Hester ${ }^{1 \dagger}$ \\ and Fadi Issa ${ }^{1 * T}$ \\ 1 Transplantation Research and Immunology Group, Nuffield Department of Surgical Sciences, University of Oxford, Oxford, \\ United Kingdom, 2 Division of Plastic Surgery, Peking Union Medical College Hospital, Peking Union Medical College and \\ Chinese Academy of Medical Sciences, Beijing, China
}

OPEN ACCESS

Edited by:

Federica Casiraghi,

Istituto di Ricerche Farmacologiche Mario Negri (IRCCS), Italy

Reviewed by:

Frans H. J. Claas,

Leiden University, Netherlands

Sofia Berglund,

Karolinska Institutet (KI), Sweden

*Correspondence:

Fadi Issa

fadi.issa@nds.ox.ac.uk

${ }^{+}$These authors have contributed equally to this work and share

senior authorship

Specialty section:

This article was submitted to Alloimmunity and Transplantation, a section of the journal

Frontiers in Immunology

Received: 04 February 2021 Accepted: 09 March 2021

Published: 25 March 2021

Citation:

Stark HL, Wang HC, Kuburic J, Alzhrani A, Hester J and Issa F (2021) Immune Monitoring for Advanced Cell

Therapy Trials in Transplantation:

Which Assays and When?

Front. Immunol. 12:664244.

doi: 10.3389/fimmu.2021.664244
A number of immune regulatory cellular therapies, including regulatory $T$ cells and mesenchymal stromal cells, have emerged as novel alternative therapies for the control of transplant alloresponses. Clinical studies have demonstrated their feasibility and safety, however developing our understanding of the impact of cellular therapeutics in vivo requires advanced immune monitoring strategies. To accurately monitor the immune response, a combination of complementary methods is required to measure the cellular and molecular phenotype as well as the function of cells involved. In this review we focus on the current immune monitoring strategies and discuss which methods may be utilized in the future.

Keywords: immune monitoring, cell therapy, transplantation, regulatory $\mathrm{T}$ cell, mesenchymal stromal cell

\section{INTRODUCTION}

The long-term treatment of transplant patients with immunosuppressive drugs is associated with significant side effects including life-threatening infections, cancer development, and direct drug toxicity (1-3). A number of immune regulatory cellular therapies including regulatory $\mathrm{T}$ cells (Tregs) and mesenchymal stromal cells (MSCs) have emerged as novel alternative therapies for the control of transplant alloresponses (4-6), with the possibility of reducing the morbidity associated with standard immunosuppression.

To date, clinical studies of advanced cellular therapies have focused on feasibility and safety. As the goal of these cellular therapies is to modify the immune response to transplantation, detailed immune monitoring in these trials is crucial. This immune monitoring facilitates a deeper understanding of the alloresponse, while providing crucial data on treatment effectiveness as well as the potential to identify new biomarkers or therapeutic targets. The immune response is a diverse and dynamic system that interacts temporospatially at many points, and it is therefore not possible (nor relevant) to monitor a single cell type in isolation. To accurately monitor the immune response, a combination of complementary methods is required to measure the cellular and molecular phenotype as well as the function of cells involved. In this review we will discuss the current methods of immune monitoring (Figure 1) and how they have the potential to become standard features of clinical trials in the future. 


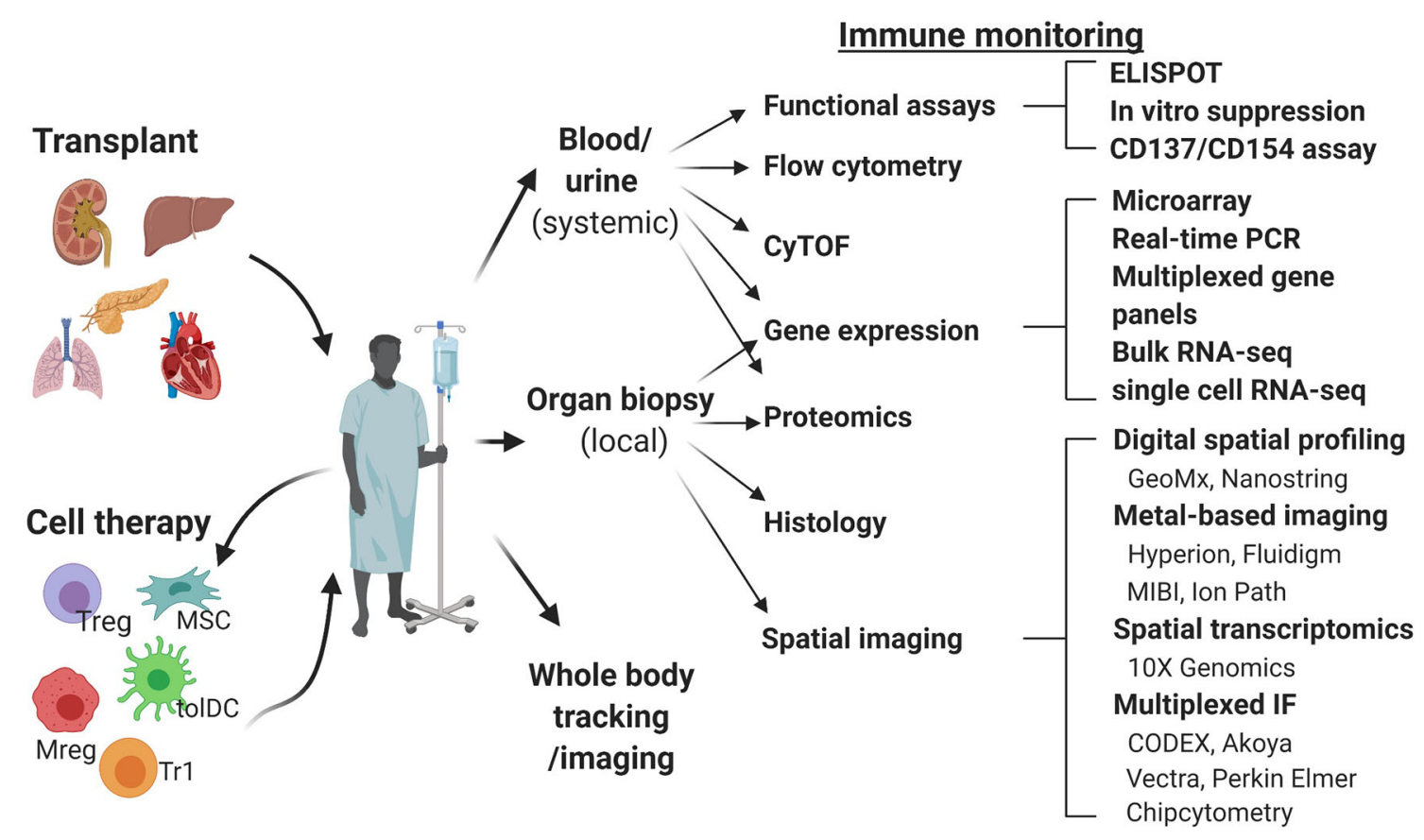

FIGURE 1 | Schematic overview of the immune monitoring methods useful in cell therapy trials in transplantation. CODEX, co-detection by indexing; CyTOF, cytometry of the time of flight; IMC, imaging mass cytometry; MIBI, multiplexed ion beam imaging; Mreg, regulatory macrophage; MSC, mesenchymal stem cell; RTqPCR, real time quantitative polymerase chain reaction; tolDC, tolerogenic dendritic cell; Treg, regulatory $T$ cell; $T r 1$, type 1 regulatory $T$ cell.

Currently, peripheral blood is the most studied material, due to the availability of well-developed techniques of analysis and ease of repeated sampling. However, data from pre-clinical models demonstrating homing of adoptively transferred human regulatory cells to the allograft and its importance for the induction of immune tolerance highlights the need for and value of allograft tissue analysis $(7,8)$. We will review here the methods most commonly used for systemic immune monitoring of blood, including flow cytometry, mass cytometry, functional assays and gene expression analysis (Figure 2) and discuss novel techniques of tissue biopsy analysis, including gene expression analysis and spatial biology methods (Figure 3).

\section{FLOW CYTOMETRY}

Flow cytometry is well established as a technique for investigating the immune response. It provides rapid multiparametric analysis of single cells in solution and is a powerful tool for immune monitoring as it can measure multiple parameters in parallel (example of data shown in Figure 2A) (9). Flow cytometers utilize lasers as light sources to produce both scattered and fluorescent light signals that are captured and converted into electronic signals (10). Cells are typically stained with fluorophore-conjugated antibodies or fluorescent dyes (10). This fluorescence is one of the limitations of the method, as the number of markers that can be analyzed concomitantly is limited by spectral overlap of the fluorophores used.
Whilst it is a commonly used method it is acknowledged that there can be significant variability in how samples are run and analyzed. This is of particular concern for clinical trials across different sites where it would be advantageous to be able to compare results. Efforts have been made to address this, Lee et al. published a consensus, the Minimum Information about a Flow Cytometry Experiment (MIFlowCyt) standard, detailing the minimum information that should be reported when publishing results of flow cytometry experiments (11), in order to aid both comparison and replication of results. Geissler et al. published the outcome of a TTS (The Transplant Society) symposium, where it was agreed it would be beneficial to establish consensus standard operating procedures (SOPs) for immune monitoring, the Virtual Global Transplantation Laboratory (vGTL) (12). To date, two SOPs, blood collection and PBMC isolation (13), and donor alloantigen specific IFN $\gamma$ ELISpot (14) have been published. Expanding on this, Cossarizza et al. published invaluable guidelines for the use of flow cytometry in immunological studies, covering in detail the various techniques and applications of flow cytometry as well as how to analyze the results (15).

The ONE study was set up to study the immune-modulatory effect of a range of different regulatory immune cells in renal transplant patients (16). A key part of the setup was the development of a rigorous immune monitoring program, to profile the peripheral blood immune phenotype, using flow cytometry. Antibody panels were developed to profile $\mathrm{T}$ cell, $\mathrm{B}$ cell and dendritic cell (DC) subsets and their activation status (now available from Beckmann Coulter, 
A

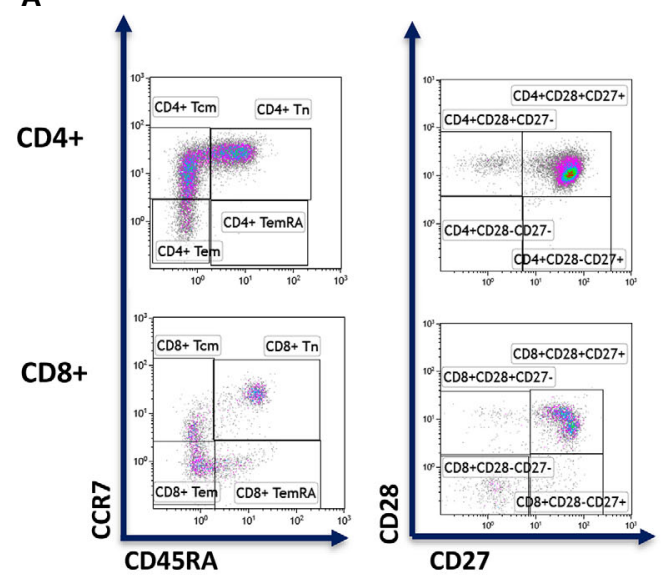

C

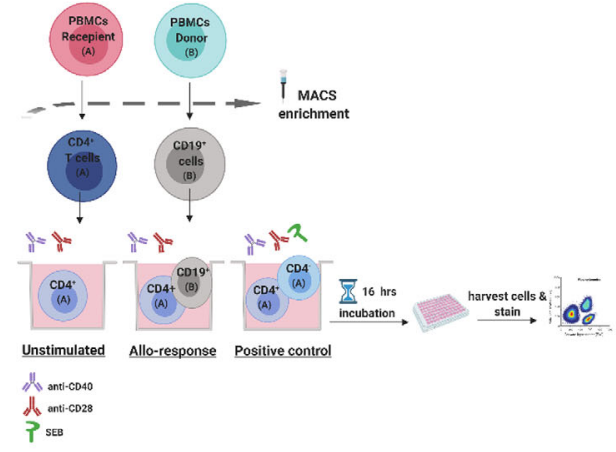

B

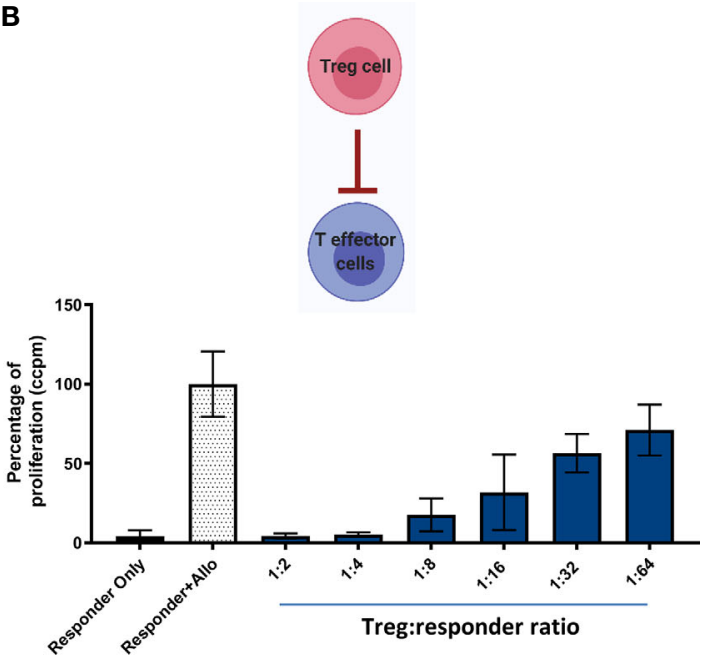

D
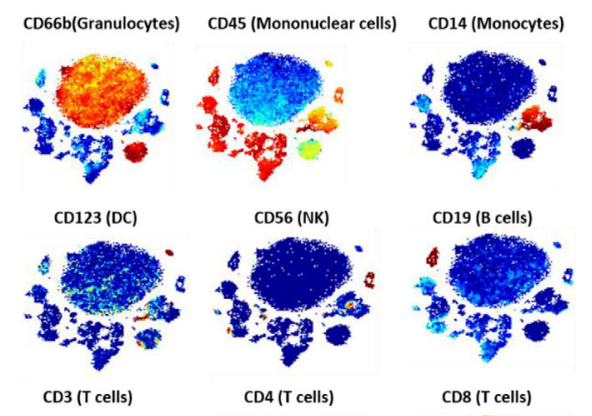

CD3 (T cells) CD4 (T cells)
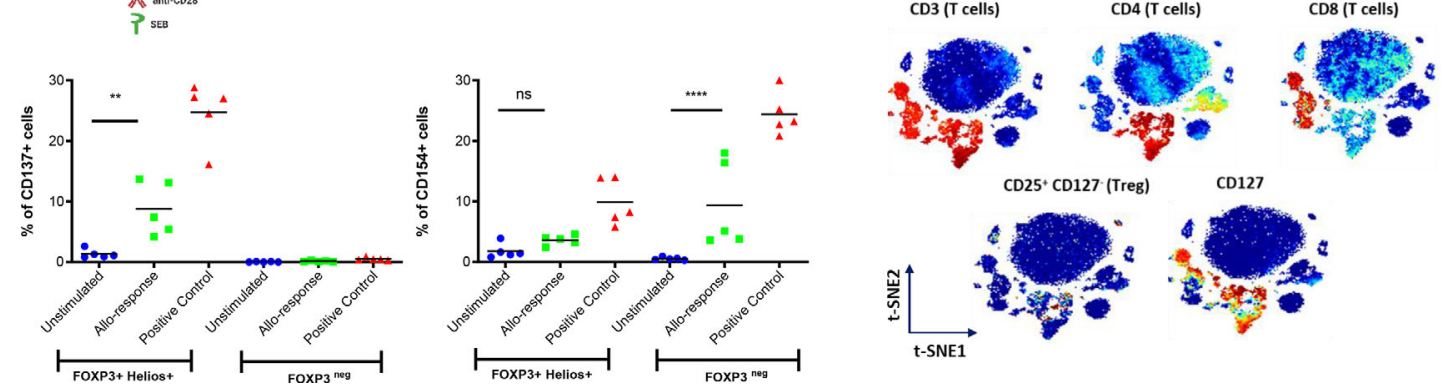

FIGURE 2 | Representative examples of systemic immune monitoring techniques. (A) Flow cytometry can be used to measure the frequency of various immune cell populations in peripheral blood. (B) In vitro suppression assays can be used to assess the suppressive potential of Tregs (or other regulatory cells). Example of $3 \mathrm{H}$ thymidine incorporation readout in a test with allogeneic stimulation. (C) (Top) Schematic of the experimental design of CD137/CD154 assay. (Bottom) Example of CD137 (left) and CD154 (right) expression on FOXP3 ${ }^{+}$Helios $^{+}$(Tregs) and FOXP3 ${ }^{\text {neg }}$ (conventional CD4+ T cells) cells. (D) Multiplexed CyTOF technology can be used for deep phenotyping analysis of leukocyte composition. An example of t-distributed Stochastic Neighbor Embedding (t-SNE) analysis of leukocyte clusters annotated based on the intensity of analyzed parameter is shown. ${ }^{\star \star}=p$ value $0.0041,{ }^{\star \star \star *}=p$ value $<0.0001, F$ test of variance. not significant $(p>0.05)$.

DURAclone panels) (17). Streitz et al. reported on the results of this optimization process, including the strategy of local sample preparation using strict standard operating procedures (SOPs), followed by central analysis. They showed acceptable variability in results between multiple international sites. Using these standardized protocols provides results that can be compared between treatment groups and patients across multiple centers, vital for immune monitoring in clinical trials (17). The same panels have been incorporated by other cell therapy trials, like the TWO study (a randomized, phase II study investigating efficacy of regulatory $\mathrm{T}$ cells in kidney transplantation) and the Neptune study (a phase I study investigating mesenchymal stromal cells in renal transplantation).

The majority of trials looking at advanced cellular therapies in transplantation have used some form of flow cytometry analysis as part of their immune monitoring $(16,18-25)$. From the more basic measurement of numbers and proportions of different immune cell subsets, to following changes in the immune 
A

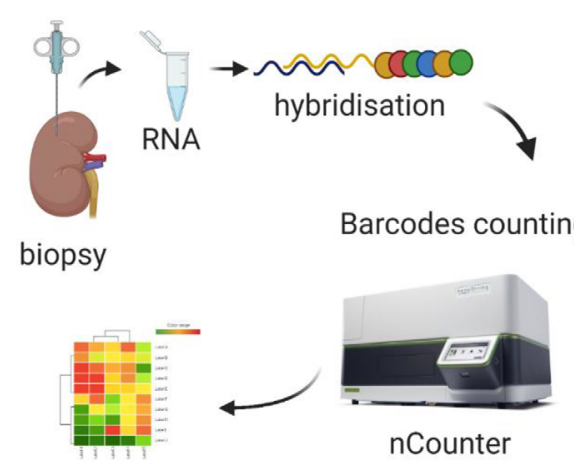

Data analysis
B

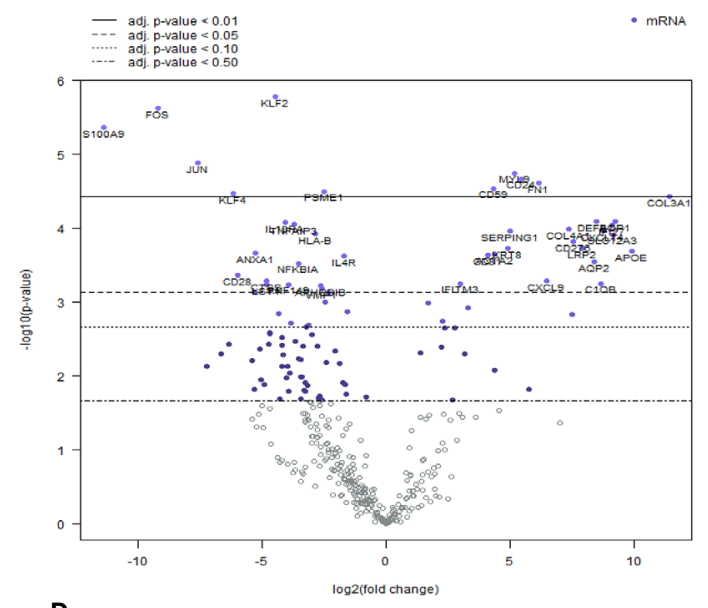

D
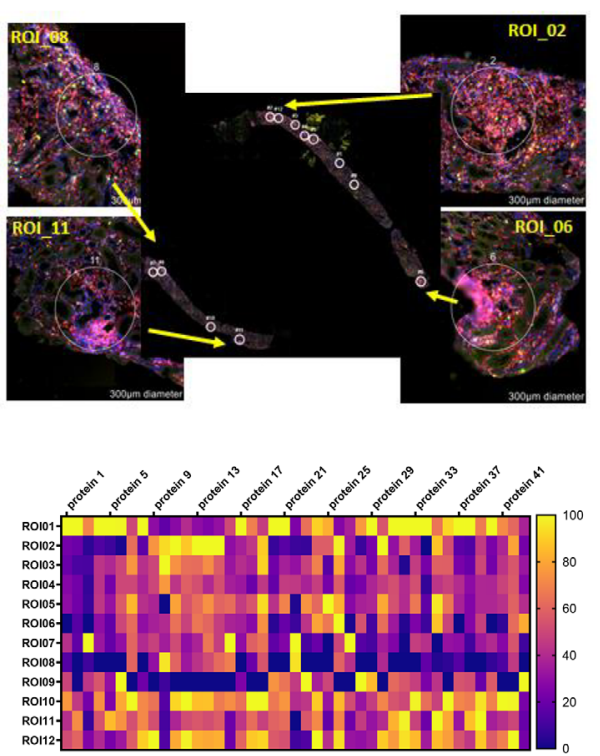

FIGURE 3 | Representative examples of the in situ immune monitoring techniques. (A) Multiplexed gene expression using the nCounter, Nanostring. Schematic of the technique. (B) An example volcano plot obtained using Banff - Human Organ Transplantation (B-HOT) panel on the nCounter system, kidney biopsy versus background of PBMC. (C) Schematic of digital spatial profiling (DSP). (D) Example of kidney biopsy from renal transplant recipient analyzed using DSP (GeoMx, Nanostring). Morphology staining, selected ROls and heat map analysis are shown. Example of geometric regions of interest (ROIs) and readout with panel of antibodies. FFPE, formalin fixed paraffin embedded; ROI, region of interest.

compartment over time. Below is a brief overview of how flow cytometry has been used for immune monitoring following cellular therapy.

\section{The ONE study}

There were six different cell based medicinal products (CBMPs) trialed in the ONE study, including polyclonal Tregs (pTregs), donor alloreactive Tregs (darTregs), autologous tolerogenic dendritic cells (ATDC) and regulatory macrophages (Mregs). The immune monitoring methods detailed by Streitz et al. (17), were utilized to allow a metanalysis of the results. There was no difference in the $\mathrm{CD} 4{ }^{+} \mathrm{CD} 25^{\text {high }} \mathrm{CD} 127^{\text {low }}$ Tregs at 60 weeks in those receiving CBMPs compared to standard care (16).
However, there was evidence of significant decreases in Treg specific demethylated region (TSDR) demethylation in the standard care group. Furthermore, there was an increase in $\mathrm{CD} 8^{+} \mathrm{T}_{\mathrm{EMRA}}$ and $\mathrm{CD} 8^{+} \mathrm{CD} 57^{+}$chronically active $\mathrm{T}$ cells in the standard care group. Both the CBMP group and standard care groups had more plasmacytoid dendritic cells at 60 weeks posttransplant than healthy age/sex matched controls. Interestingly there was a normalization of marginal zone-like $\mathrm{B}$ cell numbers and a significant reduction in $\mathrm{CD} 14^{\text {high }} \mathrm{CD} 16^{+}$monocytes in those who received a CBMP. Taken together this suggested that those who received CBMPs had restoration of an immune phenotype more similar to the healthy controls than those receiving standard of care (16). 


\section{Regulatory T cells}

In 2014 the TRACT (Treg adoptive cell therapy) trial was commenced, a phase I trial to test safety and to trial escalating doses of autologous polyclonal Treg therapy in kidney transplantation (18). Flow cytometric analysis was the main method of immune monitoring of patients in this trial. Of note, patients had induction therapy with alemtuzumab, resulting in a significant decrease in T cells as well as B cells, NK cells and $\mathrm{CD} 14^{+}$monocytes in the first month posttransplant (18). By day 90 numbers of the majority of these cells had recovered, however numbers of $\mathrm{CD} 4^{+}$and $\mathrm{CD} 8^{+} \mathrm{T}$ cells remained low (18). Interestingly, the authors observed an increase in Tregs that remained stable at 1 year (18).

Similarly, Todo et al. used flow cytometry as part of the immune monitoring strategy for patients who underwent liver transplantation combined with Treg therapy (19). They also noted a trend to increased Treg $\left(\mathrm{CD} 4{ }^{+} \mathrm{CD} 25^{+} \mathrm{CTLA}^{+} /\right.$ $\mathrm{CD}^{+} \mathrm{CD} 25^{+} \mathrm{Foxp}^{+}$) numbers post transplantation, however, they were unable to demonstrate a significant difference due to variation between recipients (19). Sánchez-Fueyo et al. used ONE study panels for immune monitoring of autologous polyclonal Treg therapy in liver transplantation. They did not find any significant changes in immune cell subsets post Treg therapy (25).

\section{Mesenchymal Stromal Cells}

The Neptune study, a phase I study of allogeneic mesenchymal stromal cells (MSCs) in kidney transplant recipients (22), utilized the same flow cytometry protocols and panels as the ONE study for immune monitoring of participants, allowing detailed monitoring of the changes in leukocyte subsets post cell therapy (22). Authors found a decrease in $\mathrm{CD} 19^{+} \mathrm{B}$ cells, $\mathrm{CD} 56^{+}$ NK cells, $\mathrm{CD} 8^{+} \mathrm{T}$ cells, $\mathrm{CD} 4^{+} \mathrm{T}$ cells and Tregs post induction immunotherapy, as expected (22). The B cells and NK cells began to recover from week 25 post induction (22). Although showing signs of repopulation, $\mathrm{T}$ cell numbers had not returned to baseline by 12 months (22). However, $\mathrm{CD} 4^{+} \mathrm{T}$ cells showed a significant increase in number after two MSC infusions (22). Later timepoint data are awaited from this trial.

Perico et al. used flow cytometry for immune monitoring of a phase I trial of autologous MSCs in renal transplantation (21). Initially they demonstrated a profound depletion of $\mathrm{CD}^{+}$and $\mathrm{CD}^{+} \mathrm{T}$ cells $(21)$. The $\mathrm{CD}^{+} \mathrm{T}$ cell numbers recovered by day 360 , however $\mathrm{CD}^{+} \mathrm{T}$ cells never regained pretransplant levels (both control and MSC group) (21). The MSC group had fewer $\mathrm{CD}^{+} \mathrm{CD} 45 \mathrm{RO}^{+} \mathrm{CD} 45 \mathrm{RA}^{-}$memory $\mathrm{T}$ cells compared to the control group by day 360 (21). They also demonstrated higher numbers of $\mathrm{CD} 4^{+} \mathrm{CD} 25^{\text {high }} \mathrm{Foxp} 3^{+} \mathrm{CD} 127^{-}$Tregs than the control group (21).

Peng et al. used flow cytometry to monitor immune response post MSC treatment in renal transplantation (20). No difference was seen between treatment and control groups in numbers of $\mathrm{CD} 4^{+} \mathrm{T}$ cells, $\mathrm{CD} 8^{+} \mathrm{T}$ cells or NK cells before or after transplant (20). However, authors did note an increased proportion of $\mathrm{CD} 27^{+}$memory B cells in the MSC treated group (20). Casiraghi et al. reported a case report of tolerance following
MSC infusion in liver transplant. They noted a high Treg: memory $\mathrm{CD}^{+} \mathrm{T}$ cells ratio, compared to control. They also demonstrated expansion in naïve and transitional B cells (26).

\section{Regulatory Dendritic Cells (DCs)}

Macedo et al. used flow cytometry for immune monitoring post allogeneic DCreg infusion in living donor liver transplant patients. They reported a decrease in $\mathrm{T}$-bet ${ }^{+}$Eomes $^{+} \mathrm{CD} 8^{+} \mathrm{T}$ cells (both central and effector memory phenotypes) following DCreg infusion (24). Conversely there was an increase in T-bet Eomes ${ }^{-} \mathrm{CD} 8^{+} \mathrm{T}$ cells of naïve phenotypes with increased $\mathrm{PD}^{+}$ and $\mathrm{Tim} 3^{+}$expression (24). Furthermore there was an increase in $\mathrm{CD} 4^{+} \mathrm{CD} 25^{+}$Foxp $^{+}{ }^{+}$Tregs, resulting in a change to the Treg : $\mathrm{CD} 8^{+}$ratio and potentially a more tolerogenic profile (24).

\section{FUNCTIONAL ASSAYS}

Functional assays have an important part to play in immune monitoring. While flow cytometry is able to provide excellent data on the phenotype of cells, functional assays provide information on what role these cells may be playing. Functional assays include measurement of cell proliferation (as an indicator of activation; an example of in vitro suppression test with proliferation readout is shown in Figure 2B), measurement of chemokines or cytokines produced by cells, or their effect on other cells (for example lysis). The limitation to these studies is that the assays often require ex vivo manipulation of some description that may not reliably replicate what is happening in vivo. Below we review the functional assays that have been used to date in clinical trials of cellular therapy.

Mathew et al. used thymidine incorporation assays to test the immunocompetence of kidney transplant patients after Treg therapy (18). They looked at recipient PBMC response to a number of antigens and mitogens including PHA, ConA and CMV (18). Immediately after transplantation responses were low, likely due to induction immunosuppression, but they were shown to gradually recover in the first year post-transplant (18). Although they did not achieve pre-transplant levels it is useful to note that there were no clinical infections recorded in this time (18). Similarly, Casiraghi et al. used mixed lymphocyte reactions to demonstrate anti-donor $\mathrm{CD}^{+} \mathrm{T}$ cell unresponsiveness following MSC transfer in liver transplant recipients, suggestive of a more tolerant profile (26).

As well as providing analysis of peripheral blood leukocyte subsets, flow cytometry may be used to look at the proliferation profile of cells. Mudrabettu et al. labeled peripheral blood mononuclear cells (PBMCs) with carboxyfluoroscein succinimidyl ester (CFSE) prior to stimulating them with anti$\mathrm{CD} 3$, anti-CD28 and IL-2. The proliferation profile of $\mathrm{CD} 4^{+} \mathrm{T}$ cells could then be measured using flow cytometry on the basis of CFSE staining (27). They demonstrated an initial decrease in proliferation in both control and treatment groups, likely secondary to immunosuppression. However, by day 90 after infusion the MSC treated group had a decrease in proliferation compared to control (27). Peng et al. used the same method but 
found no significant differences between control and MSCtreated groups (20).

The Pleximmune ${ }^{\mathrm{TM}}$ test (Plexision Inc, USA) is another functional assay that uses flow cytometry to quantify recipient $\mathrm{CD}^{+} \mathrm{CD} 45 \mathrm{RO}^{+}$memory $\mathrm{T}$ cells expressing $\mathrm{CD} 154$ after they have been cultured for 16 hours with surrogate donor PBMCs. It was used by Sánchez-Fueyo et al. to establish the donor specific alloimmune response and demonstrated hyporesponsiveness in those who had received Treg infusion (25), suggesting movement towards a more tolerogenic phenotype. It is also important to check the function of the immune cells that are being transferred to the patients. For example, CD137 $/ \mathrm{CD} 154^{-}$Tregs have been shown to be reliably associated with a stable phenotype (28). Upon a short stimulation with a relevant antigen, antigenresponsive Tregs express CD137, while antigen-responsive conventional CD4 T cells express CD154 (please see an example in Figure 2C). The CD137/CD154 assay has been used in the ONE Study to monitor the frequency of donorresponsive Tregs and conventional CD4 T cells.

Many studies have measured cytokine and chemokine levels in patient serum. Sanchez-Fueyo et al. used LEGENDplex ${ }^{\text {TM }}$ (BioLegend) to assess cytokine and chemokine (including IL-2, $-5,-12,-27$ and CXCL9 and 10) levels post infusion. In one patient who concurrently had a high fever these levels were raised, but in all other patients no significant changes were found. The LEGENDplex ${ }^{\mathrm{TM}}$ is a bead-based immunoassay that captures the soluble analyte between two antibodies, before then quantifying the amount using flow cytometry (25). Roemhild et al. utilized Luminex (another bead-based immunoassay) for assessment after autologous polyclonal Treg therapy in renal transplantation, as part of the ONE study (23), there was no change in either pro- or anti-inflammatory cytokines (TNF $\alpha$, IFN $\gamma$, IL-1, $-6,-8$ or-10. Shi et al. used ELISA to measure TGFß1 and PGE2, soluble factors released by MSCs that can modulate $\mathrm{T}$ and B cells. Both were increased at 4 weeks following MSC infusion (29). The Neptune study measured a number of cytokines and chemokines both before and 4 hours after MSC infusion (22). Using Biorad multiplexed assays they found that TNF $\alpha$ and IL-10 were both decreased following the second infusion of MSCs, a result that was maintained for the rest of the study. They did not find any significant differences in IL-4 or $\operatorname{IFN} \gamma(22)$.

Perico et al. monitored the alloimmune response to donor and third party antigen by using ELISpot assays to IFN $\gamma$, granzyme B and by cell-mediated lympholysis (21). They found that patients treated with autologous MSCs had decreased anti-donor IFN $\gamma$ memory $\mathrm{T}$ cell and anti-donor granzyme $\mathrm{B} \mathrm{CD}^{+}$cell responses compared to the control group (21). They also demonstrated a decreased cytolytic response of $\mathrm{CD}^{+} \mathrm{T}$ cells (21). Similarly, ELISpot assays have been used in the ONE Study and reported by Sawitzki et al, Roemhild et al. and Harden et al. $(16,23,30)$.

The Cylex Immuknow Assay is used to test the immune competence of a patient's T cells, by measuring the ATP synthesis of $\mathrm{CD}^{+} \mathrm{T}$ cells. Todo et al. used this as part of their immune monitoring of patients who received Treg therapy after liver transplantation (19). They were able to demonstrate results in the normal range for the majority of their participants (19).

The majority of these studies also tested for the presence of donor specific antibodies (DSAs), in particular development of de novo DSAs after transplantation $(16,18,19,22-24)$. There was not a significant increase in patients developing dnDSAs in these preliminary trials. This was the primary method for monitoring the humoral response. As discussed in section 2 (flow cytometry) immune monitoring also frequently included panels specifically to look at the B cell subsets over time post transplantation.

It would be interesting to know how the results of this immune monitoring correlate to clinical outcomes, however the studies discussed in these sections have all been early case reports or phase I trials, therefore focused on safety data and dose optimization.

\section{NEXT-GENERATION TECHNOLOGIES \\ CyTOF}

Mass spectrometry with cytometry by time of flight (CyTOF) is a key technology in multiple clinical trials where deep cellular phenotyping is important (31). In traditional flow cytometry, detection of fluorochrome-conjugated antibodies is based on wavelength in which sufficiently broad emission bands are produced. In mass cytometry, fluorescent labels are replaced with heavy metal tags that produce more narrow emission bands, as detection is based on mass. This limits signal overlap of emission spectra and facilitates an increased number of parameters to be simultaneously measured. Furthermore, these metals are not commonly found in biological specimens, reducing potential background noise (e.g. from autofluorescence) $(31,32)$.

CyTOF has the potential to overtake flow cytometry as the method of choice for immune monitoring (example of CyTOF data is shown in Figure 2B). It is possible to stain intracellularly, therefore gaining functional insights and to look at antigen specificity of $\mathrm{T}$ cells by using metal conjugated tetramers, as well as allowing a high number of cell surface markers to be concomitantly identified (31). Furthermore, analysis is unbiased, with the potential to uncover new insights into immune cell subsets. There are some limitations, it is a slow and currently expensive method in comparison to flow cytometry and cells are not available for further studies at the end of the workflow.

Sánchez-Fueyo et al. used CyTOF to characterize the Treg compartment in patients following autologous Treg infusion. They were able to identify the expanded Tregs by comparing phenotypes of individual clusters to those examined pretransfusion. They could then follow them over time, noting by one month they had mostly disappeared. The expanded Tregs were found to be more proliferative and have increased CD25, CTLA4, CD38, GATA3, PD1 and CD274 (25).

Similarly, data from a cohort of patients enrolled in the ONE Study examined how the phenotype of Tregs changed over time post transplantation using CyTOF. Distinctive alterations were observed in clustering associated with specific post- 
transplantation changes. There were significant changes in the frequency of homing markers and CCR7 ${ }^{+}$Tregs (30).

\section{Gene Expression Profiling}

Within transplantation research the focus of gene expression profiling has been to identify biomarkers of rejection or tolerance. A variety of techniques, including microarray and RT-qPCR, have been used to explore the potential mechanisms involved. However, at present gene expression profiling has not widely been used as part of the immune monitoring strategy post cellular therapy.

The potential importance of gene expression profiling in immunomonitoring has been recognized by the Banff Foundation, who created a molecular diagnostics working group to assess the available literature on this topic and plan for future research. At the latest symposium in 2019 they reached a consensus on a panel of 770 genes, to cover the innate and adaptive immune response, tolerance, rejection and infection for the monitoring of transplant patients, the Banff - Human Organ Transplant (B-HOT) panel (33). In collaboration with NanoString Technologies, this has become a commercially available panel. Of note it is possible to use with formalin fixed paraffin embedded (FFPE) samples, enabling retrospective analysis of stored samples (schematic overview of the method and an example of gene expression profiling using the B-HOT panel are shown in Figures 3A, B).

As previously highlighted, the goal of cellular therapy is to reduce the need for harmful immunosuppression by inducing a tolerogenic state in patients. Therefore, when monitoring these patients it may be beneficial to use already developed gene signatures of tolerance as a standard for comparison, such as that described by Sagoo et al. (34). Indeed this was utilized by Hutchinson et al. to monitor patients being treated with Mreg therapy (35). They used microarray platforms to compare gene expression in their patients to those of a known tolerant cohort, finding them to be similar (35). Furthermore they found that TOAG mRNA expression (known to be decreased in acute rejection) remained high in treated patients, supporting a phenotype more often seen in healthy or tolerant patients (35).

As these biomarker profiles of tolerance or rejection become validated, it is possible to imagine how they may be used as a control or comparator group for those patients who have undergone cell therapy, to gain further insights into how these therapies are affecting the immune response.

\section{Spatial Biology}

In recent years there have been significant developments in spatial profiling techniques, making this technology Nature's 2020 Method of the Year (36). In general terms these offer the possibility of extracting spatially-resolved molecular information from tissue biopsies. Whilst bulk sequencing techniques generate detailed readouts of gene expression, they potentially miss out on small differences on a cell-per-cell basis that may be significant when taken in context of the position in which they occur. These newer techniques offer the possibility to perform more in-depth, spatially guided molecular analyses of tissue biopsies, which may be particularly relevant when trying to understand the effect of cellular therapies. We will briefly consider a few of the available techniques below.

\section{Nanostring GeoMx DSP}

The GeoMx DSP enables spatially-resolved, high-plex (10s $-10,000$ s) digital quantitation of proteins and mRNA in tissue. It uses photo-cleavable oligo-tags to collect samples in a nondestructive way, whilst maintaining spatial information (37). Benefits include the direct, digital counting of mRNA or protein without the need for intervening enzyme steps, the ability to use archival FFPE samples, and the advantage that samples can be used for further downstream processing even after running through the GeoMx DSP workflow (schematic overview of the method and an example of kidney biopsy analyzed using GeoMx DSP are shown in Figures 3C, D).

\section{X Genomics}

This uses positional molecular barcodes in the cDNA synthesis reaction with an intact tissue section, before proceeding to generating a readout via RNA-seq (38). It offers the same highly spatially resolved readout as the GeoMx DSP but at present is only available for use with fresh frozen samples.

\section{Fluidigm Hyperion}

This platform works in a similar fashion to the GeoMx, but instead of oligo-tags it uses metal-conjugated antibodies, followed by laser ablation and transfer of the ablated tissue to be measured by CyTOF. It can be used with FFPE samples and can look at up to 35 different antigens at one time (39).

\section{GE Cell Dive}

This may also be used with FFPE samples or tissue microarray. After staining with dye conjugated biomarker antibodies (up to four) and collecting an image, it then uses a patented dye inactivation process to allow further staining of the same sample with different antibodies (up to 60 in total). These images are stitched together for a highly multiplexed final result (40).

\section{Codex}

This platform uses immunofluorescence technology, with an iterative workflow that uses DNA tagging technology (with capture and reporters). One of the benefits of this technology is the ability to comprehensively image the whole sample and perform unbiased cell phenotyping, rather than needed to choose regions of interest at the beginning (41).

\section{Cell Tracking}

An excellent review by Tran and Thomson covers the current state of research into the tracking of adoptively transferred cells (8). This is a significant gap in our knowledge of the mechanism of action of these cellular therapies and may provide insight into how they modulate the immune response. Ashmore-Harris et al. have reviewed in detail the principles of non-invasive cell tracking, the methods available and how they may be used to develop new cellular therapeutics (42). 
Hutchinson et al. pioneered the use of Mreg therapy in two kidney transplant patients (35). A proportion of the transfused Mregs were labeled with $45 \mathrm{Mbq}$ oxine, allowing them to track where the cells went (using SPECT). They noted that after initially settling in the lungs they went on to travel in the circulation to liver, spleen and bone marrow. None were seen in the urinary tract, suggested good survival.

Chandran et al. monitored the fate of polyclonal Treg infusion in kidney transplant patients by labeling a proportion of transferred Tregs with deuterium and then monitoring levels over time. They found a peak in number in the first week, with the labeled Tregs still present at 30 days. Numbers had fallen below the limit of detection by 3 months (43). This technique was developed by Bluestone et al. to monitor the fate of transferred polyclonal Tregs in a phase I trial in patients with type 1 diabetes (44). They also noted maximum levels of labeled Tregs at 7-14 days. Following which deuterium-Tregs decreased, to $25 \%$ of circulating Tregs at 90 days. Levels then stabilized over the next nine months. Furthermore, T effector populations did not demonstrate evidence of deuterium labeling, suggesting the transferred Tregs were stable in their identity (44).

\section{DISCUSSION}

In this paper we have reviewed the current immunomonitoring strategies used in the early and ongoing clinical trials of cellular therapy in transplantation as well as considering methods that may be of use in the future. At present the cornerstone of monitoring relies on flow cytometric analysis of peripheral blood samples to define the leukocyte subsets present. Standardized panels have been developed to enable comparison across clinical sites with good effect. These are often used in conjunction with an array of functional studies.

It is clear that the timing of sample acquisition is an important factor in any monitoring strategy. It is known that both induction and maintenance immunosuppression may have an effect on the immune cell subsets present. Indeed, a significant initial decrease in leukocytes, with slow recovery of $\mathrm{T}$ cell populations was demonstrated in a number of the studies discussed in this paper. This should be accounted for both when planning trials and reporting on the results. The ONE Study had a clear protocol for when samples were taken, together

\section{REFERENCES}

1. Daniel V, Opelz G. Clinical relevance of immune monitoring in solid organ transplantation. Int Rev Immunol (2009) 28:155-84.

2. Shipkova M, Wieland E. Editorial: Immune monitoring in solid organ transplantation. Clin Biochem (2016) 49:317-9.

3. Haidar G, Singh N. Viral infections in solid organ transplant recipients: Novel updates and a review of the classics. Curr Opin Infect Dis (2017) 30:579-88.

4. Safinia N, Grageda N, Scotta C, Thirkell S, Fry LJ, Vaikunthanathan T, et al. Cell therapy in organ transplantation: Our experience on the clinical translation of regulatory T cells. Front Immunol (2018) 9:1-8.

5. Casiraghi F, Perico N, Cortinovis M, Remuzzi G. Mesenchymal stromal cells in renal transplantation: Opportunities and challenges. Nat Rev Nephrol (2016) 12:241-53. with the same immunosuppression, allowing for a harmonized analysis across groups.

There are a growing number of new techniques that offer the potential to explore both the phenotype and function of the immune response to cellular therapies. In particular, we are now in an era of deep spatial profiling which allows us to directly analyze transplant biopsies - the principal sites of activity rather than surrogate tissues such as blood. These techniques offer the exciting possibility of discovering new tissue-specific treatment targets. However, the challenge will be in the analysis of data generated from these studies, which can be vast and open to misrepresentation. It will therefore be important to develop transparent standardized bioinformatic workflows to support the analysis and cross-site comparison of these data in order to fully understand their implications.

\section{AUTHOR CONTRIBUTIONS}

All authors contributed to the content design, literature searches, writing of the manuscript, and manuscript review. All authors contributed to the article and approved the submitted version.

\section{FUNDING}

The work from authors' own laboratory described in this manuscript have been supported by grants from Medical Research Council (MR/N027930/1), European Union Framework 7 programme though the ONE Study consortium, the Wellcome Trust $(211122 / \mathrm{Z} / 18 / \mathrm{Z})$ and Kidney Research UK (SF1/2014). Additionally, the authors acknowledge funding from the EU Horizon 2020 Research and Innovation Programme under grant agreement 825392 (RESHAPE). HS is funded by a Royal College of Surgeons Research Fellowship. AA is funded by King Abdulaziz University, Saudi Arabia. JH is a Kidney Research UK Senior Research Fellow. FI is a Wellcome Trust CRCD Fellow.

\section{ACKNOWLEDGMENTS}

Figures in this manuscript have been created using BioRender.com.

6. Kawai K, Uchiyama M, Hester J, Wood K, Issa F. Regulatory T cells for tolerance. Hum Immunol (2018) 79:294-303.

7. Issa F, Hester J, Milward K, Wood KJ. Homing of Regulatory T Cells to Human Skin Is Important for the Prevention of Alloimmune-Mediated Pathology in an In Vivo Cellular Therapy Model. PloS One (2012) 7:e53331.

8. Tran LM, Thomson AW. Detection and Monitoring of Regulatory Immune Cells Following Their Adoptive Transfer in Organ Transplantation. Front Immunol (2020) 11:1-9.

9. Maecker HT, McCoy J Jr, the FOCIS Human Immunophenotyping Consortium. A model for harmonizing flow cytometry in clinical trials. Nat Immunol (2010) 11:975-8.

10. McKinnon KM. Flow cytometry: An overview. Curr Protoc Immunol (2018) 120:5.1.1-5.1.11. 
11. Lee JA, Spidlen J, Boyce K, Cai J, Crosbie N, Dalphin M, et al. MIFlowCyt: The minimum information about a flow cytometry experiment. Cytometry Part A (2008) 73:926-30.

12. Geissler EK, Tullius SG, Chong AS. Establishment of a global virtual laboratory for transplantation: A symposium report. Transplantation (2015) 99:381-4.

13. Higdon LE, Lee K, Tang Q, Maltzman JS. Virtual Global Transplant Laboratory Standard Operating Procedures for Blood Collection, PBMC Isolation, and Storage. Transplant Direct (2016) 2:e101.

14. Carroll R, Troelnikov A, Chong AS. Virtual Global Transplant Laboratory Standard Operating Protocol for Donor Alloantigen-specific Interferongamma ELISPOT Assay. Transplant Direct (2016) 2:e111.

15. Cossarizza A, Chang H-D, Radbruch A, Acs A, Adam D, Adam-Klages S, et al. Guidelines for the use of flow cytometry and cell sorting in immunological studies (second edition). Eur J Immunol (2019) 49:1457-973.

16. Sawitzki B, Harden P, Reinke P, Moreau A, Hutchinson J, Game D, et al. Regulatory cell therapy in kidney transplantation (The ONE Study): a harmonised design and analysis of seven non-randomised, single-arm, phase 1/2A trials. Lancet (2020) 395:1627-39.

17. Streitz M, Miloud T, Kapinsky M, Reed M, Magari R, Geissler E, et al. Standardization of whole blood immune phenotype monitoring for clinical trials: panels and methods from the ONE study. Transplant Res (2013) 2:17.

18. Mathew JM, Voss J, LeFever A, Konieczna I, Stratton C, He J, et al. A Phase i Clinical Trial with Ex Vivo Expanded Recipient Regulatory T cells in Living Donor Kidney Transplants. Sci Rep (2018) 8:1-12.

19. Todo S, Yamashita K, Goto R, Zaitsu M, Nagatsu A, Oura T, et al. A pilot study of operational tolerance with a regulatory T-cell-based cell therapy in living donor liver transplantation. Hepatology (2016) 64:632-43.

20. Peng Y, Ke M, Xu L, Liu L, Chen X, Xia W, et al. Donor-derived mesenchymal stem cells combined with low-dose tacrolimus prevent acute rejection after renal transplantation: A clinical pilot study. Transplantation (2013) 95:161-8.

21. Perico N, Casiraghi F, Introna M, Gotti E, Todeschini M, Cavinato R, et al. Autologous mesenchymal stromal cells and kidney transplantation: A pilot study of safety and clinical feasibility. Clin J Am Soc Nephrol (2011) 6:412-22.

22. Dreyer GJ, Groeneweg K, Heidt S, Roelen D, van Pel M, Roelofs H, et al. Human leukocyte antigen selected allogeneic mesenchymal stromal cell therapy in renal transplantation: The Neptune study, a phase I single-center study. Am J Transplant (2020) 20:2905-15.

23. Roemhild A, Otto N, Moll G, Abou-El-Enein M, Kaiser D, Bold G, et al. Regulatory $\mathrm{T}$ cells for minimising immune suppression in kidney transplantation: Phase I/IIa clinical trial. BMJ (2020) 371:m3734.

24. Macedo C, Tran L, Zahorchak A, Dai H, Gu X, Ravichandran R, et al. Donorderived regulatory dendritic cell infusion results in host cell cross-dressing and $\mathrm{T}$ cell subset changes in prospective living donor liver transplant recipients. Am J Transplant (2020). doi: 10.1111/ajt.16393

25. Sánchez-Fueyo A, Whitehouse G, Grageda N, Cramp M, Yim T, Romano M, et al. Applicability, safety, and biological activity of regulatory $\mathrm{T}$ cell therapy in liver transplantation. Am J Transplant (2020) 20:1125-36.

26. Casiraghi F, Perico N, Gotti E, Todeschini M, Mister M, Cortinovis M, et al. Kidney transplant tolerance associated with remote autologous mesenchymal stromal cell administration. Stem Cells Trans Med (2020) 9:427-32.

27. Mudrabettu C, Kumar V, Rakha A, Yadav A, Ramachandran R, Kanwar D, et al. Safety and efficacy of autologous mesenchymal stromal cells transplantation in patients undergoing living donor kidney transplantation: A pilot study. Nephrology (2015) 20:25-33.

28. Nowak A, Lock D, Bacher P, Hohnstein T, Vogt K, Gottfreund J, et al. CD137 $+\mathrm{CD} 154$ - expression as a regulatory $\mathrm{T}$ cell (Treg)-specific activation signature for identification and sorting of stable human tregs from in vitro expansion cultures. Front Immunol (2018) 9:1-15.

29. Shi M, Liu Z, Wang Y, Xu R, Sun Y, Zhang M, et al. A Pilot Study of Mesenchymal Stem Cell Therapy for Acute Liver Allograft Rejection. Stem Cells Trans Med (2017) 6:2053-61.
30. Harden P, Game D, Sawitzki B, Van der Net J, Hester J, Bushell A, et al. Feasibility, Long-term Safety and Immune Monitoring of Regulatory T Cell Therapy in Living Donor Kidney Transplant Recipients. Am J Transplant (2020) 44:ajt.16395.

31. Hartmann FJ, Bendall SC. Immune monitoring using mass cytometry and related high-dimensional imaging approaches. Nat Rev Rheumatol (2020) 16:87-99.

32. Ornatsky O, Bandura D, Baranov V, Nitz M, Winnik M, Tanner S. Highly multiparametric analysis by mass cytometry. I Immunol Methods (2010) 361:1-20.

33. Mengel M, Loupy A, Haas M, Roufosse C, Naesens M, Akalin E, et al. Banff 2019 Meeting Report: Molecular diagnostics in solid organ transplantationConsensus for the Banff Human Organ Transplant (B-HOT) gene panel and open source multicenter validation. Am J Transplant (2020) 20: 2305-17. doi: 10.1111/ajt.16059

34. Sagoo P, Perucha E, Sawitzki B, Tomiuk S, Stephens D, Miqueu P, et al. Development of a cross-platform biomarker signature to detect renal transplant tolerance in humans. J Clin Invest (2010) 120:1848-61.

35. Hutchinson JA, Riquelme P, Sawitzki B, Tomiuk S, Miqueu P, Zuhayra M, et al. Cutting Edge: Immunological Consequences and Trafficking of Human Regulatory Macrophages Administered to Renal Transplant Recipients. J Immunol (2011) 187:2072-8.

36. Marx V. Method of the Year: spatially resolved transcriptomics. Nat Methods (2021) 18:9-14.

37. Merritt CR, Ong G, Church S, Barker K, Danaher P, Geiss G, et al. Multiplex digital spatial profiling of proteins and RNA in fixed tissue. Nat Biotechnol (2020) 38:586-99.

38. Ståhl PL, Salmen F, Vickovic S, Lundmark A, Navarro J, Magnusson J, et al. Visualization and analysis of gene expression in tissue sections by spatial transcriptomics. Science (2016) 353:78-82.

39. Giesen C, Wang H, Schapiro D, Zivanovic N, Jacobs A, Hattendorf B, et al. Highly multiplexed imaging of tumor tissues with subcellular resolution by mass cytometry. Nat Methods (2014) 11:417-22.

40. Gerdes MJ, Sevinsky C, Sood A, Adak S, Bello M, Bordwell A, et al. Highly multiplexed single-cell analysis of formalinfixed, paraffinembedded cancer tissue. Proc Natl Acad Sci United States America (2013) 110:11982-7.

41. Goltsev Y, Samusik N, Kennedy-Darling J, Bhate S, Hale M, Vazquez G, et al. Deep Profiling of Mouse Splenic Architecture with CODEX Multiplexed Imaging. Cell (2018) 174:968-81.e15.

42. Ashmore-Harris C, Iafrate M, Saleem A, Fruhwirth GO. Non-invasive Reporter Gene Imaging of Cell Therapies, including T Cells and Stem Cells. Mol Ther (2020) 28:1392-416.

43. Chandran S, Tang Q, Sarwal M, Laszik Z, Putnam A, Lee K, et al. Polyclonal Regulatory $\mathrm{T}$ Cell Therapy for Control of Inflammation in Kidney Transplants. Am J Transplant (2017) 17:2945-54.

44. Bluestone JA, Buckner JH, Fitch M, Gitelman S, Gupta S, Hellerstein M, et al. Type 1 diabetes immunotherapy using polyclonal regulatory T cells. Sci Transl Med (2015) 7(315):315ra189-315ra189.

Conflict of Interest: The authors declare that the research was conducted in the absence of any commercial or financial relationships that could be construed as a potential conflict of interest.

Copyright (c) 2021 Stark, Wang, Kuburic, Alzhrani, Hester and Issa. This is an open-access article distributed under the terms of the Creative Commons Attribution License (CC BY). The use, distribution or reproduction in other forums is permitted, provided the original author(s) and the copyright owner(s) are credited and that the original publication in this journal is cited, in accordance with accepted academic practice. No use, distribution or reproduction is permitted which does not comply with these terms. 